\title{
THE ANTI-INTUITIVE VISUAL SYSTEM OF THE HONEY BEE*
}

\author{
A. HORRIDGE** \\ Australian National University, Canberra, ACT 0200, Australia \\ (Received: November 25, 2011; accepted: December 20, 2011)
}

\begin{abstract}
Because bees fly around, visit flowers and chase mates, we conclude intuitively that they see things as we do. But their vision is unexpectedly different, so we say it is anti-intuitive. Detailed tests have demonstrated separate detectors for modulation of blue and green receptors, edge orientation (green only), and areas of black. The edge detectors are about $3^{\circ}$ across, independent, and not re-assembled to make lines, shapes or textures. Instead, the detectors of each type are summed quantitatively to form cues in each local region with an order of preference for learning the cues. Trained bees remember the positions of the total modulation (preferred), the average edge orientation, areas of black or colour, and positions of hubs of radial and circular edges in each local region, but not the original responses, so the pattern is lost. When presented with a yellow spot on a blue background with no UV reflected, the preferred cue is not the colour, but a measure of the modulation detected by the green and separately by the blue receptors.
\end{abstract}

Keywords: Honeybee - vision - discrimination - cues - memory

\section{INTRODUCTION}

As individuals, explanations of our own behaviour are almost all about our performance, i.e., what we do, and why we do it. So, efforts to analyse animal behaviour begin with the performance, long before we start to look for components and their interactions.

At the heart of the difficulties in understanding the causes of behaviour is the difference between induction and deduction. Induction has been accepted for centuries by scientists. After collecting plenty of data, a conclusion is reached intuitively, by experience and imagination that is believed to be innate and reliable. Wholly new ideas can be introduced. When there is any question of their validity, the power of reasoning and trust in the imagination are ultimately justified as derived from a rational brain that recognizes the truth. As shown by much of modern science, however, intuition is not necessarily correct.

*Presented during the 12th ISIN Symposium on Invertebrate Neurobiology, 31 August - 4 September, 2011, Tihany, Hungary.

**E-mail: horridge@netspeed.com.au 
On the other hand, the deductive method used since the days of Euclid deduced a conclusion logically from factual data combined with general truths. There was no intuition or guesswork there (so they said), but no new ideas were added. So, induction generated a sensible theory that could be validated by deduction based on the results of critical tests. Sadly, sufficient tests were often not done, so erroneous intuitive theories survived and anti-intuition was not mentioned.

We notice that bees fly around without crashing into things, visit flowers, and return if rewarded. The conclusion that the bees see the flowers and things as we do is intuitively obvious to anyone. But it is not necessarily so, and not tested.

The deduction might proceed as follows. To re-assemble the image that is divided into 90,000 cells of the retina, and to recognise even a few things, requires a very large brain. Bees have a brain of negligible size, and therefore they cannot see flowers or any object or shape as such. Also, some blind men play music, type or go shopping, as if they see quite well. Just as the performance of the blind does not prove that they can see things, we cannot make that assumption about bees.

The difficulty was noticed long ago. Felix Plateau [1] found that bees did not confuse the flowers with artificial ones no matter how accurate the colours, so he guessed that they found the reward by odour. Soon, Forel [2] repeated the experiments and also concluded that the bees did not see the flowers as we do, but they found the reward by arriving at the right place by the use of landmarks including flowers and odour. Later, there were many more discoveries but the belief that the bees see the flowers persisted.

\section{METHODS}

The apparatus (Fig. 1) and methods have been described many times (3-7). From a distance the bees have a choice between two targets subtending not more than $55^{\circ}$, so they cannot use the whole eye. Inside the apparatus the targets change sides every 5 min so the bees use only the displayed patterns to identify the place of the reward. In the current work, the ultra-violet receptors were excluded by working away from direct sunlight and by use of white or coloured papers that reflected negligible UV.

\section{RESULTS}

\section{Recognition of gratings by modulation, not orientation}

Bees quickly learn to discriminate the vertical black bars of a regular grating from the same grating with horizontal bars. With vertical versus horizontal alternating red and green bars, Turner [8] observed 90\% correct choices. Anyone looking at the targets would intuitively suppose that the bees see the bars, but it cannot be so because the brain of the bee is too small to re-assemble all those bars. We will see later that bee vision cannot even re-assemble an edge, or several edges to make a shape. 


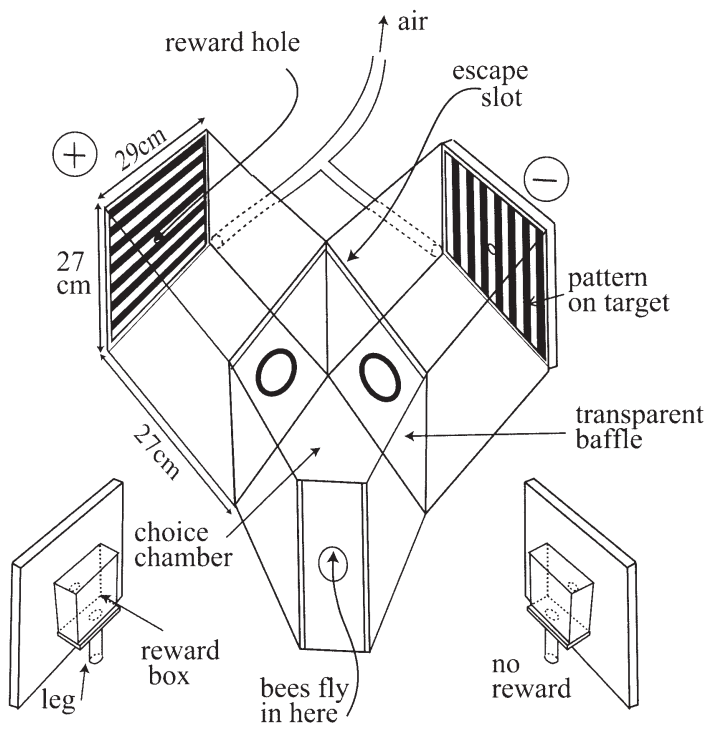

Fig. 1. The Y-choice apparatus used in all the experiments. The bees make a choice at a fixed distance from two targets. The criterion of success is when the bee passes through the hole in one of the baffles, or over the top of it. The two targets, together with the reward, change sides every $5 \mathrm{~min}$. The bees exit by the way they entered. Inset: the targets seen from behind, showing the support leg and the reward box. Redrawn with corrections, from (3-7)

(a) spontaneous preferences of untrained bees (1995)

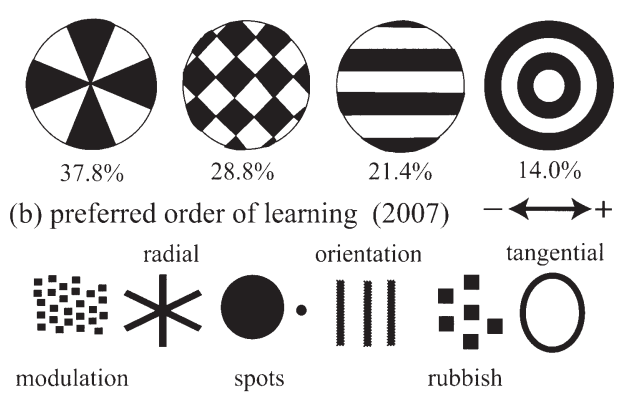

Fig. 2. (a) The spontaneous preference of untrained bees for different kinds of black patterns presented simultaneously. (b) The preferred order of learning the cues that were later discovered. Redrawn from (14) with corrections

A minimum grating period of $2^{\circ}$ was found by Hecht and Wolf [9], and near $2.5^{\circ}$ by Srinivasan and Lehrer [10] for the discrimination of vertical or horizontal gratings. The interommatidial angle of the worker bee eye was wrongly thought to be $2.5^{\circ}$ in the horizontal plane, so all authors inferred that the bees would not resolve the bars at the observed limit, so they must have discriminated the modulation that was gener- 
ated by their own relative motion, not the orientation. Later, however, it was shown that the orientation was not even the preferred cue when modulation or position was available [7].

In 1995, a group of us in Canberra published the order of preference for a variety of black and white patterns by untrained freely flying bees (Fig. 2a). Radial patterns were the most preferred, while circles were the least. Later, when the nature of the cues had been worked out, the order of preference was similar, with modulation particularly preferred but not edge orientation (Fig. 2b).

While measuring the resolution of modulation discrimination, in the mid-1990's, I rediscovered that the modulation was measured irrespective of the pattern (Fig. 3), but this astonishing tolerance towards the change in the pattern had been well described 70 years before [11]. To observe it, the variety of available cues in the training patterns must be reduced, preferably to one, which the bees detect in all the patterns.

Bees cannot discriminate two oblique orthogonal coarse gratings that display no contrast to the green receptors (Fig. 4a) because there is no difference in modulation, and the orientation detector has inputs only via the green receptors. However, the bees discriminate well between horizontal and vertical gratings with no green contrast (Fig. 4b). As shown by a simple test (Fig. 4c), they had learned the difference in the modulation irrespective of the pattern.

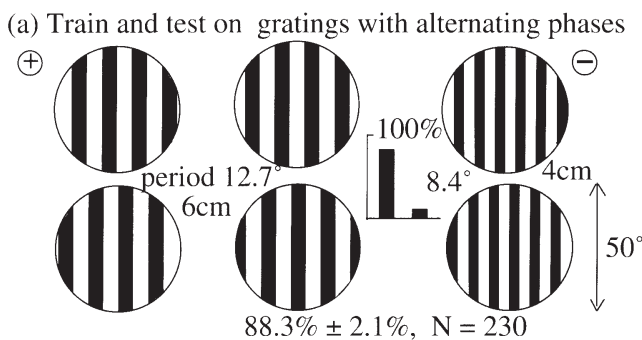

(b)Test on 6 sectors versus 8 sectors

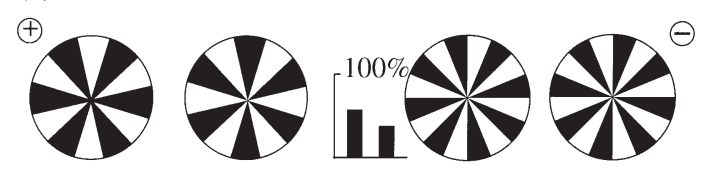

$60.1 \% \pm 4,9 \%, \mathrm{~N}=98, \mathrm{p}<0.05$

(c) Test on spirals period $10.5^{\circ}$ versus $4.2^{\circ}$

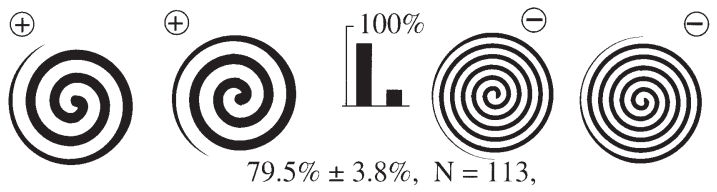

Fig. 3. The measurement of the modulation was independent of the pattern. Bees trained to discriminate the larger period of the vertical bars distinguished the larger period of sector and spiral patterns 
(a)

train with no green contrast

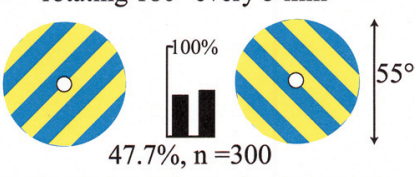

(b)

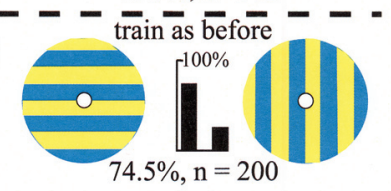

(c)

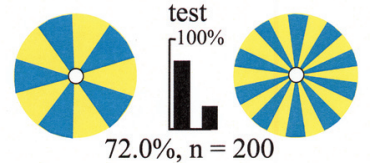

Fig. 4. The discrimination between gratings with no green contrast, with positions of areas of colour shuffled. The patterns are invisible to the green receptors. (a) Bees cannot be trained to discriminate the oblique bars, because the blue modulation is equal on the two targets. (b) When the edges are horizontal versus vertical, the bees discriminated well. (c) A test whether the trained bees learned the difference in the modulation of the blue receptors, as indeed they had. Redrawn with corrections, from (6)

It is therefore of interest to consider what the bees detected when they look at these coloured patterns. They certainly did not discriminate the patterns or the colours, and the patterns looked identical to the green receptors. The only possible conclusion is that they learned the quantitative difference in the modulation of the blue receptors displayed by each pattern, and nothing more. So, green and blue modulations are separate and effective inputs, but the pattern in colour was ignored.

\section{The edge cue and redundant positions}

Bees easily discriminated between an oblique black bar at $45^{\circ}$ and the same at $135^{\circ}$ (Fig. 5a). The preferred cues of modulation, area and position of the centre, were the same on both targets, so these cues were learned on the rewarded one but unlearned on the unrewarded one. The only available cue was the average edge orientation. The orientation was displayed equally well in thin oblique lines (Fig. 5b), but not in stepped edges (Fig. 5c), where the edges at right angles cancelled the orientation cue. The bar was not distinguished from the oblique lines (Fig. 5d), and the bars were not recognised when moved down on the targets (Fig. 5e). The logical conclusion is that the bees detected the edge orientations at the expected place, but not the bars.

On the other hand, when bees were trained on the single oblique bar at $45^{\circ}$ versus a plain blank white target (Fig. 5f), they could not distinguish it from the same bar at $135^{\circ}$ (Fig. $5 \mathrm{~g}$ ) nor from the stepped bar (Fig. 5h), and they accepted a bar at $135^{\circ}$ (Fig. 5i) and with a lower score when moved down (Fig. 5i). In this training, there- 


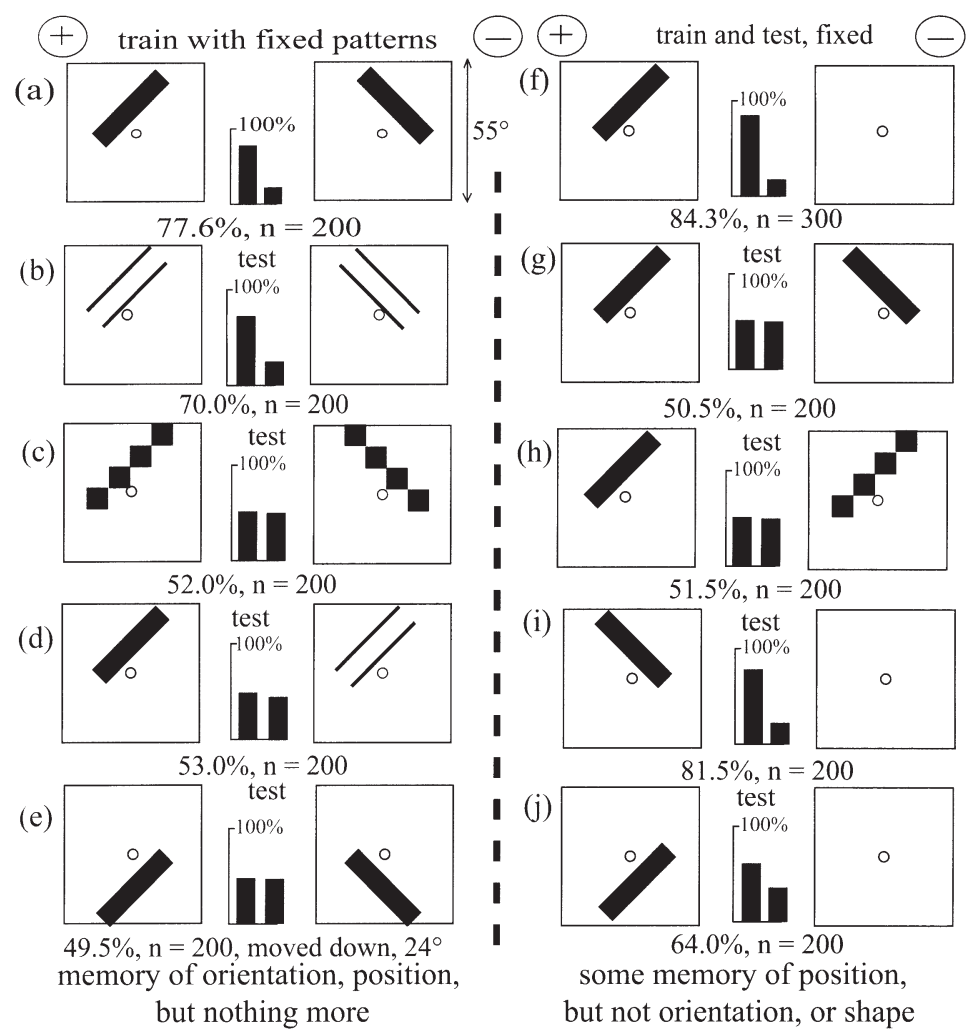

Fig. 5. With equal and orthogonal fixed black bars at corresponding places on the targets, the bees learned only the orientation of edges. (a) The training targets. (b) Edge orientation was detected. (c) The stepped bars were not discriminated because the orientation cue was eliminated by the square steps and the black areas had not been learned. (d) No discrimination between bar and edges, because the orientations were the same. (e) No discrimination when the bars were moved in the vertical direction. Redrawn with corrections, from (7). (f) Next, training with a single bar versus a blank white target. (g) No discrimination between the bar and a similar orthogonal bar. (h) No discrimination between the bar and a similar stepped bar. (i) The trained bees now accepted an orthogonal bar, and (j) the training bar that was moved down. Redrawn with corrections, from (12)

fore, the bees had learned that there was something black on the target [3], its position and a measure of the modulation, but nothing about the orientation of the bar.

\section{The size of the feature detectors}

First the bees were trained to use the orientation cue by shuffling the positions of the oblique bars to remove the cue of position (Fig. 6a). Oblique bars were used to avoid a modulation difference. The trained bees were tested with the bars broken into rows of equally spaced small squares, which cancel the orientation cue (Fig. 6b). The 

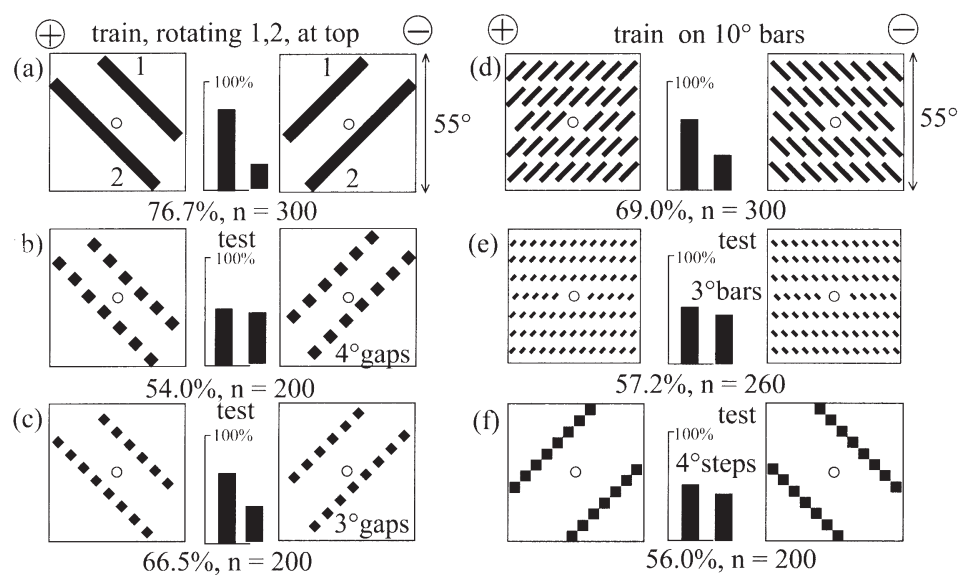

(e) $69.0 \%, \mathrm{n}=300$

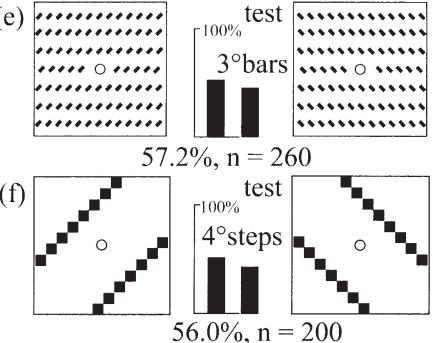

Fig. 6. The measurement of the maximum length of the detectors of edge orientation. (a) The training targets, with a constant orientation difference but positions of black areas shuffled. (b-c) Tests with the bars replaced by rows of small black squares. No orientation was detected when the squares were more than $3.5^{\circ}$ apart, because the orientation detectors were unable to bridge larger gaps. (d-f) The measurement of the minimum length of the orientation detectors. (d) The training targets, with many small parallel bars. (e) Tests with smaller bars failed when they were less than $3^{\circ}$ long. (f) With stepped bars in a test, the minimum step size was near $3.5^{\circ}$. Redrawn after (4-5)

maximum length of the orientation detector, near $3.5^{\circ}$, is shown by the minimum length of the gaps between the squares before the bees begin to detect the orientation (Fig. 6c). Therefore the detectors cannot string together to bridge across larger gaps.

To determine the minimum length of the orientation detectors, bees trained to discriminate the orientation of oblique bars (Fig. 6d) were tested with similar but smaller bars. The minimum length was near $3^{\circ}$ (Fig. 6e). When tested with oblique bars consisting entirely of square steps, the minimum step size that cancelled the orientation cue was near $3.5^{\circ}$ (Fig. 6f). The orientation detectors are therefore very short, about $3^{\circ}$ (Fig. 7).

\section{How bees distinguish the cues based on edges}

Besides the hidden preferences, the anti-intuitive visual discrimination arises from the way that the cues are formed inside the bee. The optical axes of the receptors are regularly spaced about $1^{\circ}$ apart. The receptor responses eventually converge in groups of seven on feature detectors of edge orientation and modulation (Fig. 7a-d).

We already know from the resolution of gratings that the modulation detector must consist of a very sensitive centre, with a symmetrical inhibitory surround (Fig. 7c). The edge orientation detectors are represented as similar to those used in robot vision, with integer steps of sensitivity. There must be at least three types at different orienta- 


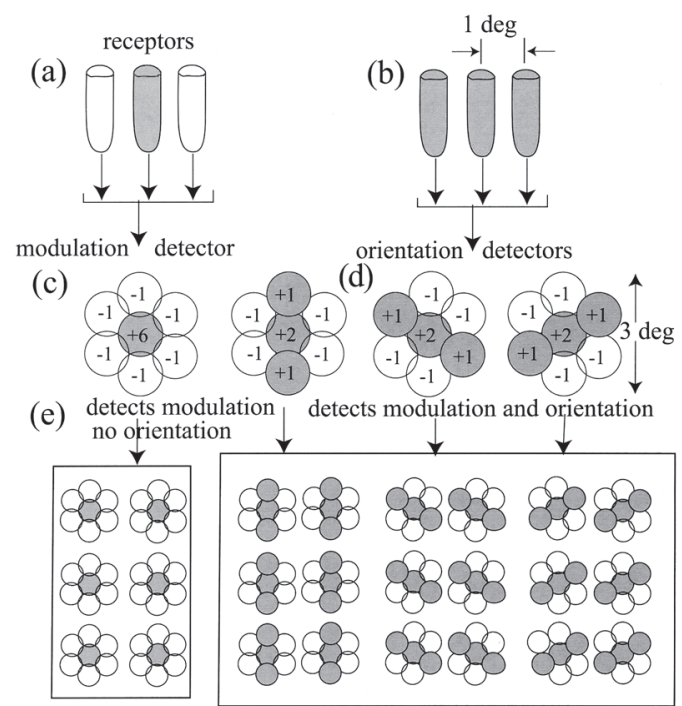

local fields, summing responses irrespective of pattern or shape

(f)

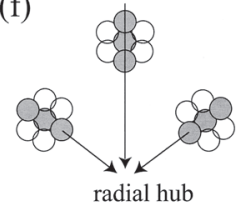

(g)

g)

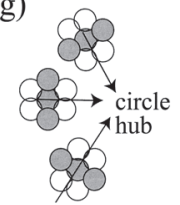

(h)

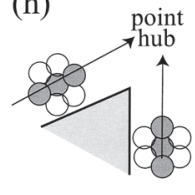

type and position of hub; modulation persists; orientation lost

Fig. 7. Components of the mechanism of measurement of cues. (a-b) Receptors. (c) Modulation detector. (d) Detectors of edge orientation. (e-h) Detector responses are summed quantitatively to form cues of (e) average edge orientation in a local region, and $(\mathrm{f}-\mathrm{h})$ identification of hubs, all irrespective of pattern. The detectors in $(\mathrm{c}-\mathrm{d})$ would be insensitive to changes of intensity. The three types of detectors of edge orientation are symmetrical about a line, so they cannot distinguish between white/black and black/white at an edge. The cues detect the types of pattern and are quantitative summations irrespective of pattern. Redrawn from (14)

tions, because edges at any orientations can be distinguished. Bees can distinguish the orientation of an edge where the light/dark and the dark/light sides alternate every 5 min, but cannot tell which side of an edge is darker or lighter. Therefore the orientation detectors must be symmetrical about a line. A separate mechanism detects the position of the centre and size of areas of colour or black irrespective of the positions of the edges.

In the next step, the responses of each type of feature detector are summed quantitatively in each local region of the eye to yield a scalar quantity of the modulation and of the length of edge. The summation of all the responses in Figure 7e preserves only the positions of the totals and the average orientation in the local region, but the individual responses and therefore the pattern, are lost. 


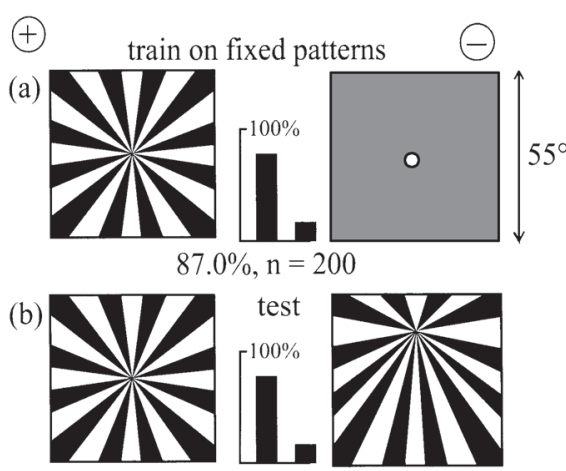

$88.0 \%, \mathrm{n}=200$

(c)
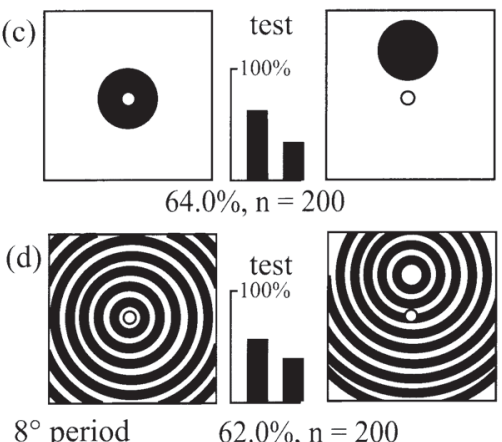

Fig. 8. When trained on a radial or circular pattern, the bees learned the position of the hub in the vertical direction, with an accuracy of about $5^{\circ}$, irrespective of the pattern. (a) Training versus $50 \%$ grey. (b) Tests with various shifts in the hub position. (c-d) Tests with quite different patterns, showing that the position of the hub is an independent cue. Redrawn after (15)

The size of the local region was measured in experiments in which edges at different angles or in different positions were allowed to cancel the orientation cue (Fig. $5 \mathrm{c}, 6 \mathrm{f}$ ). The bee does not remember the pattern, only the strengths and positions of cues. Moreover, the average orientation may be zero but the total modulation is still a cue.

At the same level in the processing, three types of hubs are identified at the positions of the centre of parts of a circular structure, a radial or spokes pattern, and of a corner (Fig. $7 \mathrm{f}-\mathrm{h}$ ). Tests show that the position and kind of the hub can be learned, irrespective of the actual pattern (Fig. 8) and even when the symmetry is incomplete.

When trained on a radial or circular pattern (Fig. 8a), the bees learned the position of the hub in the vertical direction, with an accuracy of about $5^{\circ}$. Tests with various shifts in the hub position show that it is an independent cue, irrespective of the pattern (Fig. 8b-d). The centre of an area of black also acts as a hub. 


\section{Interchange of two colours with no green contrast}

When bees were trained on a target with a yellow and a blue $20^{\circ}$ spot on a grey background, with no contrast to the green receptors, they failed to learn (Fig. 9a) because green contrast is essential for motion perception, so the eyes are not stabilized on the target. However, when black spokes were added to each target they learned very well, even with spots down to $6^{\circ}$ (Fig. 9b). The effect was immediate; the colours had not changed but the bees were now able to discriminate. Their behaviour suggested that they were able to fixate on the centre, with one spot projected to each eye. Moreover, the trained bees were able to discriminate the spots alone without the spokes (Fig. 9c), showing that they had learned to use other landmarks within the apparatus to stabilize the eye on a target with no green contrast. A vertical line was equally effective (Fig. 9d). Of course, these experiments do not prove that they learned even one colour, because they may have learned only the position of the blue modulation.

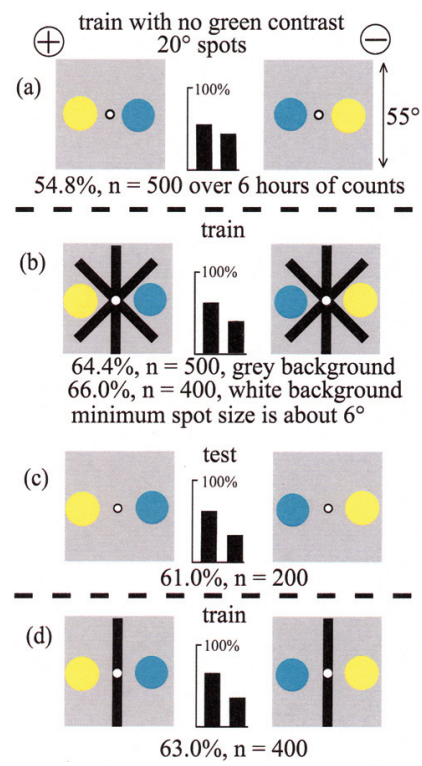

Fig. 9. The motion perception system requires green contrast, so bees cannot be trained to discriminate the positions of two coloured discs with no green contrast because the eye cannot be stabilised on the target. (a) Training patterns. (b) With spokes added to stabilise the eye, the minimum spot size is near $6^{\circ}$. (c) After training on (b) the trained bees discriminate the spots. Because the coloured spots stimulate the green receptors equally, the bees use the blue receptors to discriminate the locations, as in Fig. 10. (d) A vertical bar is equally good to stabilize the eye. Redrawn after (13) 


\section{Failure to remember the colour}

One would suppose that bees trained to a coloured spot would remember its colour, but experiments prove otherwise. To simplify the experiment, the ultra-violet receptors were put out of action by working in the shade and by use of coloured papers that reflected negligible UV.

The bees were trained to discriminate between a yellow $20^{\circ}$ spot on a blue background versus a plain blue target, with no contrast to the green receptors (Fig. 10a). There was a large area of blue on each target, so the blue colour would be a poor cue. The two inputs that they had available were the tonic detection of yellow photons and the modulation of the blue receptors; i.e., the local reduction in the blue photon flux and the local blue modulation in the local region of the eye corresponding to the spot.

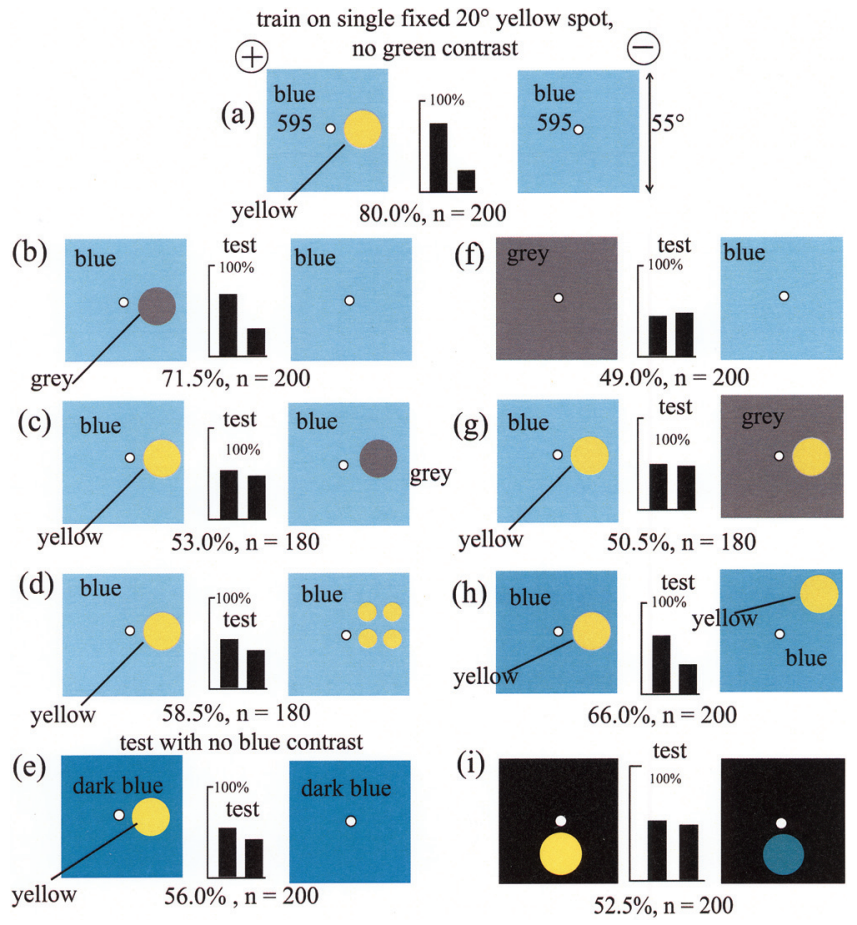

Fig. 10. When trained on targets with no green contrast, bees used a measure of the modulation of the blue receptors, not the colours. (a) The bees were trained to a $20^{\circ}$ yellow spot on a blue background, with no green contrast, versus a plain blue target. (b) They responded strongly to a grey spot (55\% black) with no green contrast, so they did not require the yellow colour. (c) They confused the yellow spot with a grey spot with the same modulation of the blue receptors. (d) They could just discriminate the spot from 4 small spots of the same colour. (e) They responded poorly to the spot with no blue contrast. (f) They had not learned to avoid the plain blue target. $(\mathrm{g})$ They did not recognize the training target versus one with the same modulation pattern. (h) They had learned the position of the spot. (i) A black background spoils the training 
In place of the yellow spot, the trained bees accepted a grey one with similar modulation (Fig. 10b). For the same reason they were unable to distinguish between the yellow spot and a grey one with no green contrast (Fig. 10c). In fact, a spot of any colour would serve as long as the modulation in that local eye region matched that in the training. Clearly, they had not learned the yellow colour. This result makes nonsense of the usual definition of colour vision and the test for it in bees. The suggestion that the grey spot on a blue background looked yellow is a restatement of the observation and not an explanation at all. So, next we must look for what they had detected.

The trained bees could just distinguish the yellow spot from four smaller yellow spots of the same total area at the corresponding place (Fig. 10d), as if they noticed a difference in the modulation of the blue receptors. They gave an insignificant response to the spot displayed with no blue contrast, on a background of darker blue (Fig. 10e). They had not learned to avoid the plain blue target (Fig. 10f) and failed to recognise the training pattern versus the yellow spot on a grey background with no green contrast (Fig. 10g). They distinguished the training pattern from the same with the spot moved up (Fig. 10h), and failed to discriminate between the yellow and blue spots on a black background (Fig. 10i). Taken together, these tests show that the trained bees had learned a measure of the modulation of the blue receptors, with little indication of detection of colour.

In the training, therefore, the first preference of the bees was to learn to go to the place of blue modulation, not the colour yellow. Like the experiments of Hess [12], the above results suggest that many previous experiments should be reconsidered. The preferred cue was a measure of the available modulation, which in this example was the blue receptor modulation.

\section{With no blue contrast}

A similar experiment with no blue contrast gave a similar result (Fig. 11). The blue colour was darker than before, giving less contrast to the blue receptors, and the yellow was also different. The trained bees had not learned to avoid the unrewarded target, but had lost their preference for blue (Fig. 11b). They accepted a grey spot, with no blue contrast, in place of the yellow one (Fig. 11c), and they did not recognize a yellow spot with no green contrast (Fig. 11d) because they relied on the green receptor modulation. The trained bees did not recognize the training pattern when presented versus the yellow spot on a green background (Fig. 11e), nor a yellow spot with no blue contrast versus a grey one, both on the blue background (Fig. 11f). They did, however respond to the grey spot with no blue contrast versus a black one, both on the blue background (Fig. 11g). In each case, the result is compatible with learning a measure of green contrast. Finally, the smallest resolvable yellow spot with no blue contrast subtended about $5^{\circ}$ (Fig. 11h).

The bees had clearly not learned the yellow colour, or the unrewarded target, and there was no blue contrast, so they must have learned to go to the expected measure of the modulation of the green receptors. 


\section{More doubts about colour discrimination}

Many more experiments are needed to answer all the questions raised by the tests in Figures 10 and 11. Length of training was an important variable. When bees were trained to discriminate between a $20^{\circ}$ spot that alternated every 5 min between yellow and blue on a white background versus a plain white target, at first they learned nothing, but after $6 \mathrm{~h}$ training they performed quite well (Fig. 12a). However, the trained bees then accepted a grey spot as well as either of the coloured ones (Fig. 12b). They did not recognise either colour, so they had learned to accept anything as preferable to nothing, or to avoid the blank target (Fig. 12c, d).

When trained with a yellow versus a blue $20^{\circ}$ spot on a black background, bees did not learn the obvious difference in $3 \mathrm{~h}$ of training (Fig. 12e). The reason is not clear; the colours were clearly visible but possibly the modulation was too far from the expected range.

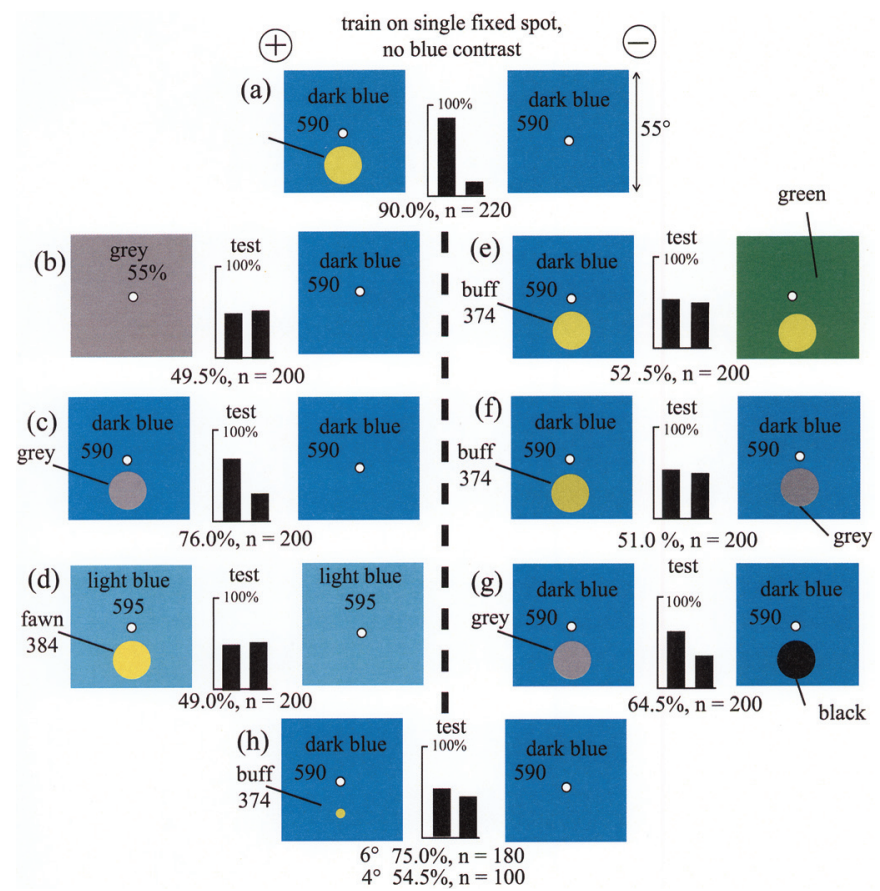

Fig. 11. When trained on targets with no blue contrast, bees used a measure of the modulation of the green receptors, not the colours. (a) The bees were trained to a $20^{\circ}$ buff yellow spot on a dark blue background, with no blue contrast, versus a plain blue target. (b) They had not learned to avoid the plain blue target. (c) They responded to a grey spot (55\% black) with no blue contrast, so they did not need the yellow colour. (d) They responded poorly to the spot with blue contrast but no green contrast. (e) They could not recognize the training pattern versus the spot on a green background, or (f) from a white spot on a blue background. (g) Given a grey versus a black spot, they selected the one with familiar green contrast. (h) The minimum spot size was about $5^{\circ}$ 
As previously observed with black spots or bars, bees easily learned to discriminate the position of a coloured spot on a white background, if the alternative positions were on the same side of the targets (Fig. 12f) but not if they were on opposite sides (Fig. 12g), suggesting that they fixated on the centre of the target as a reference point to learn the spot's position. Elsewhere it has been concluded that the bees recognize cues with the region of the eye that did the learning [13].

When trained with two different coloured spots, one above the other, on the same side of the target (Fig. 12h), the bees learned the rewarded positions of the two colours separately (Fig. 12i, j). We cannot conclude, however, that with one eye the bees can remember two different colours because the critical experiments have not been done. The position and quantity of the blue modulation would have been sufficient.

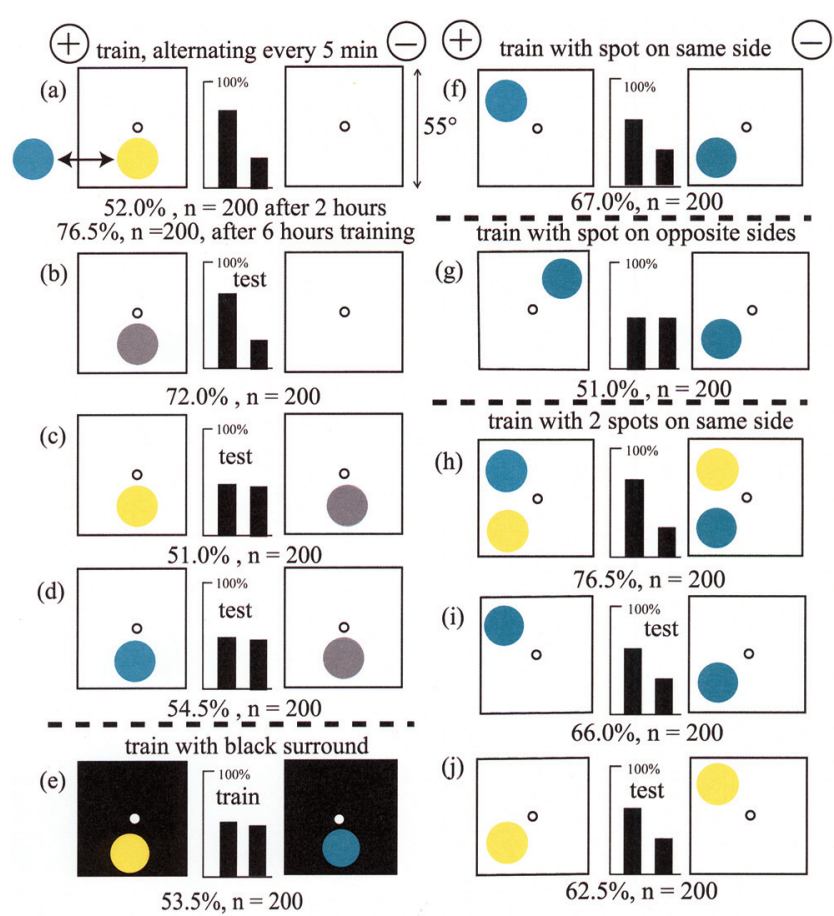

Fig. 12. (a) When trained on a $20^{\circ}$ spot that alternated between blue and yellow every 5 or $10 \mathrm{~min}$, on a white background, versus a plain white target, the bees at first learned nothing, but improved after $6 \mathrm{~h}$ of training. (b) In a test with a grey spot, the trained bees showed that they had not learned the colour. (c, d) The trained bees could not tell the difference between the grey spot and either of the coloured spots in the training. (e) When trained with a black surround, they learned nothing. (f, g) The bees discriminated the position in the vertical direction when the spots were on the same side of the target, but not on opposite sides. (h) They discriminated the two spots from the same inverted. (i,j) They remembered the separate spots at the training positions 


\section{DISCUSSION}

\section{The mechanism of colour vision}

We already know that the position and a quantitative measure of the modulation in each local region of the visual field is the preferred visual cue for black and white patterns [14]. Electrophysiology has demonstrated neural machinery that would explain detection of the modulation at each type of receptor. The retinula cells adapt rapidly to changes in intensity; the lamina ganglion cells adapt even faster. The largefield neurons of the deep optic lobe are mostly phasic and reveal a great variety of antagonistic inputs from two or three receptor types, weighted in different proportions so that they detect modulation in different parts of the spectrum. The neuron properties are more suitable to detect line-labelled modulation than a tonic, maintained photon flux. None of that seems to be adapted to the discrimination of colour as a palette of persistent sensations. Moreover, within the optic lobe there is no sign of the colour triangle, or of neurons that could discriminate between colours. The spatial fields for colour-coded neurons are large, which implies that they are adapted to quantitative measurement of a summed mixture, region by region, but not the identification of the original inputs.

The new results imply that much old work will have to be revised, for example the effect of the colour of the illumination upon another colour, and the discrimination of large areas of colour. The part played by the UV receptors is little understood.

Humans cannot help categorizing pattern and colour, but the preferred processing is quite different in bees. They detect the coincidences of modulated responses of many large-field neurons with antagonistic inputs. This is not the end of the story, however, because when modulation is not available, as in large uniform surfaces or illumination from a beam of light, bees may discriminate something that we call colour.

These results also make us wonder what the bees actually detect when they fly around. There was nothing to show that bees have anything more than feature detectors and innate circuitry to recognize a place by the coincidences of cues [14). The visual input is combined with many other senses that are active all the time to build up a total impression of the adjacent environment, which we humans might appreciate as the way that a bat or a blind man is aware of the surroundings. Whenever the bees' behaviour suggests a higher function graced by an abstract noun, such as cognitive behaviour, or detection of abstract properties such as shape, similarity, symmetry, colour in general, or texture, we have simply looked for the appropriate tests to demonstrate the actions of feature detectors and cues.

\section{REFERENCES}

1. Plateau, F. (1885-1897 in 5 parts) Comment les fleurs attirent les insects. Recherches expérimentales. Bull. Acad. Roy. Soc. Belg., 30-34. See also papers listed by Forel (1908) p.142.

2. Forel, A. (1908) The Senses of Insects. Translated by M. Yearsley. Methuen, London. 
3. Horridge, G. A. (2003) Discrimination of single bars by the honeybee (Apis mellifera). Vision Res. 43, $1257-1271$

4. Horridge, G. A. (2003) The visual system of the honeybee (Apis mellifera): the maximum length of the orientation detector. J. Insect Physiol. 49, 621-628.

5. Horridge, G. A. (2003) Visual resolution of the orientation cue by the honeybee (Apis mellifera). J. Insect Physiol. 49, 1145-1152.

6. Horridge, G. A. (2003) Visual resolution of gratings by the compound eye of the bee (Apis mellifera). J. Exp. Biol. 206, 2105-2110.

7. Horridge, G. A. (2007) The preferences of the honeybee (Apis mellifera) for different visual cues during the learning process. J. Insect Physiol. 53, 877-889.

8. Turner, C. H. (1911) Experiments on pattern vision of the honeybee. Biol. Bull. 21, 249-264.

9. Hecht, S., Wolf, E. (1929) The visual acuity of the honeybee. J. Gen. Physiol. 12, 727-760.

10. Srinivasan, M. V., Lehrer, M. (1988) Spatial acuity of honeybee vision, and its spectral properties. J. Comp. Physiol. A 162, 159-172.

11. Hertz, M. (1933) Über figurale Intensität und Qualitäten in der optische Wahrnehmung der Biene. Biologische Zentralblatte 53, 10-40.

12. Hess, C. (1918) Beiträge zur Frage nach einem Farbensinne bei Bienen. Archiv für die gesapte Physiol. 170, 337-366.

13. Horridge, G. A. (1999) Pattern vision of the honeybee (Apis mellifera): The effect of pattern on the discrimination of location. J. Comp. Physiol. A 185, 105-113.

14. Horridge, G. A. (2009) What does the honeybee see? And how do we know? A critique of scientific reason. ANU ePress, Canberra, p. 360. http://epress.anu.edu.au/honeybee citation.html

15. Horridge, G. A. (2006) Visual discrimination of spokes, sectors, and circles by the honeybee (Apis mellifera). J. Insect Physiol. 52, 984-1003.

16. Horridge, G. A. (2006) Some labels that are recognized on landmarks by the honeybee (Apis mellifera). J. Insect Physiol. 52, 1254-1271. 